\title{
A relationship between semiclassical and centroid correlation functions
}

\author{
Qiang Shi and Eitan Geva \\ Department of Chemistry and The FOCUS Center, University of Michigan, Ann Arbor, \\ Michigan 48109-1055
}

(Received 3 December 2002; accepted 10 February 2003)

\begin{abstract}
A general relationship is established between semiclassical and centroid-based methods for calculating real-time quantum-mechanical correlation functions. It is first shown that the linearized semiclassical initial-value-representation (LSC-IVR) approximation can be obtained via direct linearization of the forward-backward action in the exact path integral expression for the correlation function. A Kubo-transformed two-time correlation function, with the position operator as one of the two operators, is then cast in terms of a carefully crafted exact path integral expression. Linearization of the corresponding forward-backward action, supplemented by the assumption that the dynamics of the centroid is decoupled from that of the higher normal modes, is then shown to lead to the centroid correlation function. (c) 2003 American Institute of Physics.
\end{abstract}

[DOI: $10.1063 / 1.1564814]$

\section{INTRODUCTION}

Most of the measurable quantities in condensed-phase systems can be expressed in terms of real-time correlation functions. ${ }^{1-6}$ In many cases, classical mechanics provide a reasonable approximation within which to calculate these correlation functions. However, there are many situations where this is not the case. Important examples include: (1) Rates of chemical reactions that involve light particles, such as protons and electrons, where tunneling and zero-point energy effects play a major role; (2) Energy relaxation of high frequency vibrations, where the dominant degrees of freedom (DOF) involved are not in the classical limit; (3) Optical response functions, whose temporal behavior reflects the time evolution of an electronic superposition state, which lacks a well defined classical limit.

The exact calculation of real-time quantum-mechanical correlation functions for general many-body systems remains far beyond the reach of currently available computer resources, due to the exponential scaling of the computational effort with the number of $\mathrm{DOF}^{7}$ The challenge therefore translates into the development of effective, yet computationally feasible and versatile, approximate methods for calculating quantum-mechanical real-time correlation functions. Several such methods have been proposed throughout the years, including mixed quantum-classical approaches, ${ }^{8-13}$ the analytical continuation method, ${ }^{14-21}$ Centroid Molecular Dynamics (CMD), ${ }^{22-46}$ and methods based on the semiclassical (SC) approximation. ${ }^{7,47-65}$ These methods have been applied, with relative success, to a rather extensive set of systems. However, the relationship, if any, between the various approximations underlying different methods is often not clear, and comparisons based on specific applications may be misleading.

In this paper, we develop a general relationship between the centroid and SC methodologies. The question that we address is as follows: What assumptions are required in order to obtain the centroid correlation function from the SC cor- relation function? The remainder of this paper is organized as follows. Section II provides an overview of the centroid and SC methodologies, with emphasis on results particularly relevant to the goal of this paper. A direct derivation of the linearized SC initial-value representation (LSC-IVR) approximation from the exact real-time path integral expression for a correlation function is presented in Sec. III. The relationship between centroid and LSC-IVR correlation functions is established in Sec. IV. The main results and conclusions are summarized and discussed in Sec. V.

\section{AN OVERVIEW OF THE CENTROID AND SEMICLASSICAL METHODOLOGIES}

\section{A. The centroid approach}

In its most recent formulation, ${ }^{28,29}$ centroid dynamics has been shown to be based on the following phase-space operator (given here in 1D, for simplicity):

$$
\hat{\phi}\left(x_{c}, p_{c}\right)=\frac{\hbar}{2 \pi} \int_{-\infty}^{\infty} d \xi \int_{-\infty}^{\infty} d \eta e^{i \xi\left(\hat{x}-x_{c}\right)+i \eta\left(\hat{p}-p_{c}\right)-\beta \hat{H}},
$$

where $x_{c}$ and $p_{c}$ are the centroid position and momentum, respectively, and $\beta=1 / k_{B} T$ is the inverse temperature. A central role is reserved for the trace of this operator, which corresponds to the centroid density:

$$
\rho_{c}\left(x_{c}, p_{c}\right)=\operatorname{Tr}\left[\hat{\phi}\left(x_{c}, p_{c}\right)\right] .
$$

The centroid approach also associates a classical-like centroid symbol, $A_{c}\left(x_{c}, p_{c}\right)$, with each quantum dynamical observable, $A(\hat{x}, \hat{p})$, which is defined by:

$$
A_{c}\left(x_{c}, p_{c}\right)=\operatorname{Tr}\left[\hat{\phi}\left(x_{c}, p_{c}\right) \hat{A}\right] / \rho_{c}\left(x_{c}, p_{c}\right) .
$$

The centroid density, $\rho_{c}\left(x_{c}, p_{c}\right)$, turns out to have a classical-like form, which is similar to that of the classical Boltzmann distribution:

$$
\rho_{c}\left(x_{c}, p_{c}\right)=e^{-\beta p_{c}^{2} / 2 m} e^{-\beta V_{c m}\left(x_{c}\right)} .
$$


TABLE I. The centroid formulation of quantum statistical mechanics.

\begin{tabular}{|c|c|c|}
\hline & Standard & Centroid \\
\hline$Z=$ & $\operatorname{Tr}\left(e^{-\beta \hat{H}}\right)$ & $\iint \frac{d x_{c} d p_{c}}{2 \pi \hbar} e^{-\beta\left[p_{c}^{2} / 2 m+V_{c m}\left(x_{c}\right)\right]}$ \\
\hline$\langle\hat{A}\rangle=$ & $\operatorname{Tr}\left(e^{-\beta \hat{H}} \hat{A}\right) / Z$ & $\iint \frac{d x_{c} d p_{c}}{2 \pi \hbar} e^{-\beta\left[p_{c}^{2} / 2 m+V_{c m}\left(x_{c}\right)\right]} A_{c}\left(x_{c}, p_{c}\right) / Z$ \\
\hline$C_{B A}^{\mathrm{Kubo}}(t)=$ & $\int_{0}^{\beta} \frac{d \lambda}{\beta} \operatorname{Tr}\left(e^{-\beta \hat{H}} \hat{B} \hat{A}(t+i \lambda / \hbar)\right)$ & $\frac{d x_{c} d p_{c}}{2 \pi \hbar} e^{-\beta\left[p_{c}^{2} / 2 m+V_{c m}\left(x_{c}\right)\right]} B_{c}\left(x_{c}, p_{c}\right) A_{c}\left[x_{c}, p_{c} ; t\right]$ \\
\hline
\end{tabular}

$V_{c m}\left(x_{c}\right)$ in Eq. (4) is called the centroid potential. It is distinctly different from the classical potential and can be written in terms of a constrained imaginary-time path integral:

$$
\begin{aligned}
e^{-\beta V_{c m}\left(x_{c}\right)} \equiv & \rho_{c}\left(x_{c}\right) \\
= & \left(\frac{2 \pi \beta \hbar^{2}}{m}\right)^{1 / 2} \int_{x(0)=x(\beta \hbar)} D x(\tau) \\
& \times \delta\left[x_{c}-(\beta \hbar)^{-1} \int_{0}^{\beta \hbar} d \tau x(\tau)\right] \\
& \times \exp \{-\mathcal{S}[x(\tau)] / \hbar\} \\
= & \lim \left(\frac{2 \pi \beta \hbar^{2}}{m}\right)^{1 / 2}\left(\frac{m P}{2 \pi \beta \hbar^{2}}\right)^{P / 2} \\
& P \rightarrow \infty \\
& \times \int d x_{1} \cdots \int d x_{P} \delta\left(x_{c}-\frac{1}{P} \sum_{k=1}^{P} x_{k}\right) \\
& \times \exp \left\{-\mathcal{S}\left[x_{1}, \ldots, x_{P}\right] / \hbar\right\},
\end{aligned}
$$

with

$$
\begin{aligned}
\frac{1}{\hbar} \mathcal{S}[x(\tau)] & =\lim _{P \rightarrow \infty} \frac{1}{\hbar} \mathcal{S}\left[x_{1}, \ldots, x_{P}\right] \\
& =\frac{1}{\hbar} \int_{0}^{\beta \hbar} d \tau\left[\frac{1}{2} m[\dot{x}(\tau)]^{2}+V[x(\tau)]\right]
\end{aligned}
$$

and

$$
\begin{aligned}
\frac{1}{\hbar} \mathcal{S}\left[x_{1}, \ldots, x_{P}\right]= & \beta\left\{\sum_{k=1}^{P} \frac{m P}{2 \beta^{2} \hbar^{2}}\left(x_{k}-x_{k+1}\right)^{2}\right. \\
& \left.+\frac{1}{P} \sum_{k=1}^{P} V\left(x_{k}\right)\right\} .
\end{aligned}
$$

In Eq. (7), $x_{P+1}=x_{1}$. It should be noted that $\rho_{c}\left(x_{c}\right)$ is proportional to the probability density of finding a classical cyclic chain polymer consisting of $P$ beads, which are connected by harmonic springs and subject to the potential $V(x) / P$, with their center of mass (the centroid) at $x=x_{c}$. The centroid also corresponds to the zero-frequency normal mode of the chain polymer. The imaginary-time path integral in Eq. (5) can be computed using classical molecular dynamics or Monte Carlo simulations (PIMD and PIMC, respectively) for relatively complex many-body systems. ${ }^{66,67}$
The above definitions form the basis for an exact classical-like formulation of quantum statistical mechanics, which is summarized in Table I. The last line in Table I is of particular importance since it relates the classical-like twotime centroid correlation function with the exact Kubotransformed quantum-mechanical correlation function. It should be noted that Kubo-transformed correlation functions can be converted to regular correlation functions via a well known identity. ${ }^{30}$ However, this relationship is subject to two limitations: (1) It is exact only when $\hat{B}$ is linear in $\hat{x}$ and/or $\hat{p} ;(2)$ The exact time dependence of the centroid symbol $A_{c}\left[x_{c}, p_{c} ; t\right]$ is given by:

$$
A_{c}\left(x_{c}, p_{c} ; t\right)=\operatorname{Tr}\left[\hat{\phi}_{c}\left(x_{c}, p_{c}\right) e^{i \hat{H} t / \hbar} \hat{A} e^{-i \hat{H} t / \hbar}\right] / \rho_{c}\left(x_{c}, p_{c}\right),
$$

and requires the same amount of effort to calculate as in standard quantum mechanics.

The second difficulty can be circumvented by introducing computationally feasible approximations for the dynamics of the centroid symbol. Several such approximations have been proposed. ${ }^{29}$ However, they all have one important feature in common-the centroid is assumed to move on an effective potential, obtained by averaging over the higher normal modes of the imaginary-time path. Hence, the centroid is effectively decoupled from the higher normal modes. For example, the CMD method, which is by far the most popular centroid-based method, involves the following approximation: ${ }^{29}$

$$
B_{c}\left[x_{c}, p_{c} ; t\right] \approx B_{c}\left[x_{c}(t), p_{c}(t)\right]
$$

where $x_{c}(t)$ and $p_{c}(t)$ are propagated as classical-like position and momentum variables on the centroid potential, $V_{c m}\left(x_{c}\right)$ [cf. Eqs. (4) and (5)]. An important feature of the CMD approximation is that it reduces to the exact result in the following limits: (1) At short times (exact at $t=0$ ); (2) At the classical limit; (3) For harmonic systems.

Finally, it is important to note that quantum corrections are introduced into centroid methods in two distinctively different ways:

- Via the nonclassical sampling of the initial centroid positions from $\rho_{c}\left(x_{c}\right)$, rather than from the classical Boltzmann distribution. 
- The possibly nonclassical dynamics of the centroid symbols, which depends on the approximation employed (e.g., classical-like propagation on the centroid potential in the case of CMD).

\section{B. The semiclassical approach}

SC methods ${ }^{7,47-65}$ are generally based on performing a stationary-phase (saddle-point) approximation on the quantum time-evolution operator, $e^{-i \hat{H} t / \hbar} \cdot{ }^{68-76}$ The resulting SC propagator is given in terms of an integral, over classical trajectories, of $e^{i S / \hbar}$, where $S$ is the classical action. Most recent work in this area has been based on the initial-valuerepresentation (IVR) of the semiclassical propagator, which puts it in terms of an integral over initial positions and momenta. ${ }^{50-53,64,77-84}$ Different IVRs can be introduced, based on the choice of basis set. ${ }^{51,79}$ For example, the SCIVR approximation of the propagator in terms of the position eigenstates, given here for a 1D system, for simplicity, is:

$$
\begin{aligned}
e^{-i \hat{H} t / \hbar} \approx & (2 \pi i \hbar)^{-1 / 2} \int d x_{0} \int d p_{0}\left|\frac{\partial x_{t}\left(x_{0}, p_{0}\right)}{\partial p_{0}}\right|^{1 / 2} \\
& \times e^{i S_{t}\left(x_{0}, p_{0}\right) / \hbar}\left|x_{t}\right\rangle\left\langle x_{0}\right| .
\end{aligned}
$$

A straightforward application of the SC-IVR methodology to the calculation of a two-time correlation function yields:

$$
\begin{aligned}
\operatorname{Tr}\left(\hat{A} e^{i \hat{H} t / \hbar} \hat{B} e^{-i \hat{H} t / \hbar}\right) \approx & (2 \pi i \hbar)^{-1} \int d x_{0}^{+} \int d p_{0}^{+} \int d x_{0}^{-} \\
& \times \int d p_{0}^{-}\left|\frac{\partial x_{t}^{+}}{\partial p_{0}^{+}}\right|^{1 / 2}\left|\frac{\partial x_{t}^{-}}{\partial p_{0}^{-}}\right|^{1 / 2} \\
& \times\left\langle x_{0}^{+}|\hat{A}| x_{0}^{-}\right\rangle\left\langle x_{t}^{-}|\hat{B}| x_{t}^{+}\right\rangle e^{i\left(S_{t}^{+}-S_{t}^{-}\right) / \hbar},
\end{aligned}
$$

where $x_{t}^{+}=x_{t}\left(x_{0}^{+}, p_{0}^{+}\right), x_{t}^{-}=x_{t}\left(x_{0}^{-}, p_{0}^{-}\right), S_{t}^{+}=S_{t}\left(x_{0}^{+}, p_{0}^{+}\right)$ and $S_{t}^{-}=S_{t}\left(x_{0}^{-}, p_{0}^{-}\right)$are calculated along classical trajectories. The main problem involved in the evaluation of this integral has to do with the highly oscillatory nature of the integrand (different initial conditions lead to different values of the action). Early attempts to deal with this difficulty were based on filtering or stationary-phase Monte Carlo methods. ${ }^{85-88}$ However, these techniques are difficult to extend to problems involving many DOF, ${ }^{89,90}$ although the performance may be improved in some cases by using a generalized Filinov transformation. ${ }^{91}$ Another problem has to do with the pre-exponential factors which tend to grow very rapidly in chaotic systems.

Several approaches have been suggested over the last few years in order to overcome the above mentioned difficulties. One idea is based on the assumption that the most important contributions to the integral come from forward and backward trajectories which are infinitesimally close to each other. If so, one can expand the forward-backward action, $S_{t}^{+}-S_{t}^{-}$, to first order with respect to the difference between the forward and backward positions and momenta. ${ }^{47,48,56,92-97}$ The resulting linearized SC-IVR (LSCIVR) approximation then assumes the following form:

$$
\begin{aligned}
& \operatorname{Tr}\left(\hat{A} e^{i \hat{H} t / \hbar} \hat{B} e^{-i \hat{H} t / \hbar}\right) \\
& \quad \approx(2 \pi \hbar)^{-1} \int d x_{0} \int d p_{0} A_{W}\left(x_{0}, p_{0}\right) B_{W}\left(x_{t}^{(C l)}, p_{t}^{(C l)}\right),
\end{aligned}
$$

where

$$
A_{W}(x, p)=\int d \Delta e^{-i p \Delta / \hbar}\langle x+\Delta / 2|\hat{A}| x-\Delta / 2\rangle
$$

is the Wigner transform of $\hat{A},{ }^{98}$ and $\left\{x_{t}^{(C l)}\right.$ $\left.=x_{t}^{(C l)}\left(x_{0}, p_{0}\right), p_{t}^{(C l)}=p_{t}^{(C l)}\left(x_{0}, p_{0}\right)\right\}$ are propagated classically with the initial condition $x_{0}$ and $p_{0}$. Thus, the problematic pre-exponential factor is eliminated in this approximation, and the oscillatory character of the integrand is "hidden" in the Wigner transform. The major advantage of LSC-IVR has to do with its computational feasibility (although the computation of the Wigner transform in systems with many DOF is not trivial ${ }^{47}$ ). Its main disadvantage has to do with the fact that it can only capture dynamical coherent effects that arise from short-time interferences between the various trajectories (the longer time dynamics is purely classical). ${ }^{92}$

Another approach is based on the forward-backward (FB) procedure. ${ }^{57,58,60-62,64,90,99-101}$ The basic idea is to combine the forward and backward propagations involved in $e^{i \hat{H} t / \hbar} \hat{B} e^{-i \hat{H} t / \hbar}$ into a single time propagation, and apply the SC-IVR approximation to it. The resulting expression for the correlation function involves a single phase-space average over a far less oscillatory integrand (due to partial selfcancellations in the FB action), and a smaller pre-exponential factor. The implementation of the FB-IVR approach is straightforward when the operator $\hat{B}$ can be written in terms of a complex exponential. The application of the FB approach to non-exponential operators is based on expressing them in terms of exponential operators. Unfortunately, this procedure is not unique, and different representations can lead to different results. ${ }^{59,90,102}$

The different SC-IVR methods mentioned above were successfully applied in recent years to a wide array of important problems, including tunneling and interference effects in chemical reactions; ${ }^{47,58,82,83,92,103}$ nonadiabatic dynamics; $; 3,94,104,105$ photodissociation; ${ }^{101,106-108}$ the calculation of the vibrational-rotational energy levels $;{ }^{89}$ threshold photodetachment spectra ${ }^{109}$ absorption, emission, and resonant Raman spectra of $I_{2}$ in rare gas matrices ${ }^{54,55,63}$ and clusters; ${ }^{61}$ anharmonic vibrational dynamics, ${ }^{110}$ and more. These successful applications strongly suggest that SC-IVR can effectively account for most important quantum aspects in MD simulations.

\section{Contact points between CMD and LSC-IVR}

The centroid approach is based on the rational that realtime information can be obtained from computationally feasible imaginary-time calculations, while the SC approach is based on the rational that the real-time calculation can be simplified to the point where it becomes computationally feasible. Thus, at first sight, CMD and SC-IVR seem to be 
rather different. However, a closer inspection reveals several intriguing similarities, particularly between LSC-IVR and CMD. In actual applications, one is usually interested in calculating a canonical correlation function of the form $\operatorname{Tr}\left(e^{-\beta \hat{H}} \hat{A} e^{i \hat{H} t / \hbar} \hat{B} e^{-i \hat{H} t / \hbar}\right)$. The LSC-IVR approximation would then amount to performing the initial sampling based on the Wigner transform of $e^{-\beta \hat{H}} \hat{A}$, followed by fully classical dynamics of the Wigner symbol of $\hat{B}$. CMD also involves nonclassical sampling, which is based on the centroid distribution, rather than the Wigner distribution, and is followed by classical-like dynamics of the centroid symbol of $\hat{B}$ on the centroid potential. Furthermore, it was recently shown by Jang and Voth ${ }^{28}$ that the Wigner transform of the Boltzmann operator, $e^{-\beta \hat{H}}$, is given by the trace of the following phase space operator:

$$
\hat{\phi}^{\prime}(x, p)=\frac{\hbar}{2 \pi} \int_{-\infty}^{\infty} d \xi \int_{-\infty}^{\infty} d \eta e^{i \xi(\hat{x}-x)+i \eta(\hat{p}-p)} e^{-\beta \hat{H}},
$$

which is elusively similar to (and yet distinctly different from!) the centroid phase-space operator $\hat{\phi}(x, p)$ in Eq. (1), whose trace gives the centroid distribution. It should also be noted that both LSC-IVR and CMD yield the exact result at the same limits (classical limit, harmonic systems, and at $t$ $=0)$. Finally, it is also of interest to compare the performance of CMD and LSC-IVR in specific benchmark problems. We are only aware of one example in the literature where LSC-IVR and CMD have been applied to the same multi-dimensional benchmark problem. This example corresponds to the calculation of barrier crossing rate constants in a symmetrical double-well bilinearly coupled to a harmonic bath. ${ }^{45,47,111}$ In this case, both CMD and LSC-IVR performed rather well, and yielded comparable results.

The above mentioned "contact points" between CMD and LSC-IVR provided the motivation for the work reported below. Our basic objective in the following sections would be to establish a rigorous relationship between LSC-IVR and CMD, that will clarify the nature of the relationship between these two methods.

\section{A DIRECT DERIVATION OF THE LINEARIZED SEMICLASSICAL INITIAL-VALUE-REPRESENTATION (LSC-IVR) APPROXIMATION}

In this section we present a direct derivation of the LSCIVR approximation, which is based on the linearization of the exact real-time path integral expression for the correlation function. More specifically, we show that it is possible to derive LSC-IVR without explicitly invoking the SC approximation (although one can argue that linearization represents a stronger approximation which implicitly implies the SC approximation). Another goal of this section is to demonstrate the basic strategy that will be employed in the following section in order to relate LSC-IVR and centroid correlation functions.

Consider the following general two-time correlation function:

$$
C_{A B}(t)=\operatorname{Tr}\left(\hat{A} e^{i \widehat{H t} / \hbar} \hat{B} e^{-i \widehat{H t} / \hbar}\right) .
$$

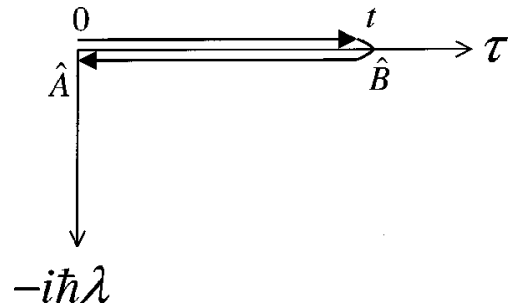

FIG. 1. A schematic view of the real-time contour underlying the path integral of Eq. (16). The imaginary time axis is included for reference only.

As is well known, $C_{A B}(t)$ can be expressed in terms of the following real-time path integral [written below in terms of the discrete time, $\{0, \epsilon, 2 \epsilon, \ldots, N \epsilon=t\}$ (the limit $N \rightarrow \infty$ will be deferred to a later stage)]:

$$
\begin{aligned}
C_{A B}(t)= & \int d x_{0}^{+} \int d x_{0}^{-} \int d x_{N}^{+} \int d x_{N}^{-}\left\langle x_{0}^{+}|\hat{A}| x_{0}^{-}\right\rangle \\
& \times\left\langle x_{0}^{-}\left|e^{i \hat{H} t / \hbar}\right| x_{N}^{-}\right\rangle\left\langle x_{N}^{-}|\hat{B}| x_{N}^{+}\right\rangle\left\langle x_{N}^{+}\left|e^{-i \hat{H} t / \hbar}\right| x_{0}^{+}\right\rangle \\
= & \left(\frac{m}{2 \pi \hbar \epsilon}\right)^{N} \int d x_{0}^{+} \cdots \int d x_{N}^{+} \int d x_{0}^{-} \ldots \\
& \times \int d x_{N}^{-}\left\langle x_{0}^{+}|\hat{A}| x_{0}^{-}\right\rangle\left\langle x_{N}^{-}|\hat{B}| x_{N}^{+}\right\rangle e^{i\left(S_{N}^{+}-S_{N}^{-}\right) / \hbar} .
\end{aligned}
$$

The forward and backward actions are given by

$$
S_{N}^{ \pm}=\sum_{j=0}^{N-1} \epsilon\left[\frac{1}{2} m\left(\frac{x_{j+1}^{ \pm}-x_{j}^{ \pm}}{\epsilon}\right)^{2}-V\left(x_{j}^{ \pm}\right)\right],
$$

where $\epsilon=t / N$. The forward-backward "time contour" underlying the path integral in Eq. (15) is shown in Fig. 1.

In the next step, we change the integration variables from $x_{0}^{+}, \ldots, x_{N}^{+}, x_{0}^{-}, \ldots, x_{N}^{-}$into $y_{0}, \ldots, y_{N}, z_{0}, \ldots, z_{N}$, such that

$$
y_{j}=\frac{1}{2}\left(x_{j}^{+}+x_{j}^{-}\right), \quad z_{j}=x_{j}^{+}-x_{j}^{-} .
$$

The linearization approximation is introduced by expanding the forward-backward action, $S_{N}^{+}-S_{N}^{-}$, to first order in $z_{0}, \ldots, z_{N}$. This yields:

$$
\begin{aligned}
S_{N}^{+}-S_{N}^{-} \approx & \epsilon \sum_{j=0}^{N-1}\left[\frac{m}{\epsilon^{2}}\left(y_{j+1}-y_{j}\right)\left(z_{j+1}-z_{j}\right)-V^{\prime}\left(y_{j}\right) z_{j}\right] \\
= & \epsilon \sum_{j=1}^{N-1} z_{j}\left[\frac{m}{\epsilon^{2}}\left(2 y_{j}-y_{j-1}-y_{j+1}\right)-V^{\prime}\left(y_{j}\right)\right] \\
& +\epsilon z_{0}\left[-\frac{m}{\epsilon^{2}}\left(y_{1}-y_{0}\right)-V^{\prime}\left(y_{0}\right)\right] \\
& +\epsilon z_{N} \frac{m}{\epsilon^{2}}\left(y_{N}-y_{N-1}\right) .
\end{aligned}
$$

Following the linearization, one can perform the integration over $z_{1}, \ldots, z_{N-1}$ explicitly, by using the following identity: 


$$
\begin{aligned}
& \int d z_{j} e^{-i / \hbar \epsilon\left[m / \epsilon^{2}\left(y_{j+1}-2 y_{j}+y_{j-1}\right)+V^{\prime}\left(y_{j}\right)\right] z_{j}} \\
& =\frac{2 \pi \hbar}{\epsilon} \delta\left[\frac{m}{\epsilon^{2}}\left(y_{j+1}-2 y_{j}+y_{j-1}\right)+V^{\prime}\left(y_{j}\right)\right] .
\end{aligned}
$$

It should also be noted that in the limit $N \rightarrow \infty(\epsilon \rightarrow 0)$,

$$
\begin{aligned}
& \boldsymbol{\epsilon} z_{0}\left[-\frac{m}{\epsilon^{2}}\left(y_{1}-y_{0}\right)-V^{\prime}\left(y_{0}\right)\right] \rightarrow-z_{0} p_{0}, \\
& \boldsymbol{\epsilon} z_{N} \frac{m}{\epsilon^{2}}\left(y_{N}-y_{N-1}\right) \rightarrow z_{N} p_{N},
\end{aligned}
$$

where $p_{0} / m=\lim _{\epsilon \rightarrow 0}\left(y_{1}-y_{0}\right) / \epsilon$ and $p_{N} / m=\lim _{\epsilon \rightarrow 0}\left(y_{N}\right.$ $\left.-y_{N-1}\right) / \epsilon$. Changing the integration variables $y_{1}, \ldots, y_{N-1}$ into $f_{1}, \ldots, f_{N-1}$, such that

$$
f_{j}=\frac{m}{\epsilon^{2}}\left(y_{j+1}-2 y_{j}+y_{j-1}\right)+V^{\prime}\left(y_{j}\right),
$$

and explicitly integrating over $f_{1}, \ldots, f_{N-1}$, then leads to the following approximation:

$$
\begin{aligned}
C_{A B}(t) \approx & \frac{1}{2 \pi \hbar} \int d y_{0} \int d y_{t} \int d z_{0} \int d z_{t}\left|\frac{\partial p_{0}}{\partial y_{t}}\right| \\
& \times\left\langle y_{0}+z_{0} / 2|\hat{A}| y_{0}-z_{0} / 2\right\rangle \\
& \times\left\langle y_{t}-z_{t} / 2|\hat{B}| y_{t}+z_{t} / 2\right\rangle e^{-i p_{0} z_{0} / \hbar} e^{i p_{t} z_{t} / \hbar} .
\end{aligned}
$$

It should be noted that in arriving to Eq. (23), we have explicitly incorporated the limit $N \rightarrow \infty(\epsilon \rightarrow 0)$, such that $y_{N}$ $\rightarrow y_{t}$ and $z_{N} \rightarrow z_{t}$, and made use of the following identity ${ }^{112}$

$$
\lim _{N \rightarrow \infty} \frac{1}{\epsilon}\left(\frac{m}{\epsilon^{2}}\right)^{N-1}\left|\frac{\partial y}{\partial f}\right|=\frac{1}{m}\left|\frac{\partial p_{0}}{\partial y_{t}}\right| .
$$

$(|\partial y / \partial f|$ is the determinant of the $(N-1) \times(N-1)$ matrix whose $(i, j)$ th element is $\left.\partial y_{i} / \partial f_{j}\right)$. It should also be noted that $y_{t}=y_{t}\left(y_{0}, p_{0}\right)$ in Eq. (23) follows a classical trajectory [cf. Eq. (20)]:

$$
\begin{aligned}
f_{j} & =\frac{m}{\epsilon^{2}}\left(y_{j+1}-2 y_{j}+y_{j-1}\right)+V^{\prime}\left(y_{j}\right)=0 \\
& N \rightarrow \infty \\
& \rightarrow m \frac{d^{2}}{d t^{2}} y(t)=-V^{\prime}[y(t)] .
\end{aligned}
$$

Finally, changing the integration variable $y_{t}$ into $p_{0}$, which amounts to a transformation to the IVR, and noting the definition of the Wigner transform [cf. Eq. (13)], yields the LSC-IVR approximation [cf. Eq. (12)]:

$$
C_{A B}(t) \approx(2 \pi \hbar)^{-1} \int d y_{0} \int d p_{0} A_{W}\left(y_{0}, p_{0}\right) B_{W}\left(y_{t}, p_{t}\right),
$$

where $y_{t}=y_{t}\left(y_{0}, p_{0}\right)$ and $p_{t}=p_{t}\left(y_{0}, p_{0}\right)$ follow a classical trajectory. Thus, we obtained LSC-IVR by direct linearization of the exact path integral expression, Eq. (16), and without explicitly invoking the SC approximation. Although we did not explicitly invoke the SC approximation, we will continue to refer to the approximation embodied in Eq. (26) as the LSC-IVR approximation.

\section{A RELATIONSHIP BETWEEN THE LSC-IVR AND CENTROID CORRELATION FUNCTIONS}

In this section we will focus our attention on the following Kubo-transformed correlation function:

$$
C_{A x}^{\mathrm{Kubo}}(t)=\frac{1}{\beta} \int_{0}^{\beta} d \lambda \operatorname{Tr}\left(e^{-(\beta-\lambda) \hat{H}} \hat{A} e^{-\lambda \hat{H}} e^{i \hat{H} t / \hbar} \hat{x} e^{-i \hat{H} t / \hbar}\right)
$$

[we refrained from including the factor $1 / Z$ in the definition of $C_{A x}^{\text {Kubo }}(t)$ since it does not play an active role in the following analysis]. It should be noted that this type of correlation function can be directly related to the corresponding centroid correlation function. In fact, it has been shown that the latter is identical to $C_{x A}^{\mathrm{Kubo}}(t)=C_{A x}^{\mathrm{Kubo}}(-t)$, provided that the dynamics of the centroid $x_{c}$ is exact. ${ }^{28}$ A straightforward application of the linearization approximation of Sec. III to $C_{A x}^{\text {Kubo }}(t)$ will yield the following approximation:

$$
\begin{aligned}
C_{A x}^{\mathrm{Kubo}}(t) \approx & \frac{1}{\beta} \int_{0}^{\beta} d \lambda \int d x_{0} \int d p_{0} \\
& \times\left(e^{-(\beta-\lambda) \hat{H}} \hat{A} e^{-\lambda \hat{H}}\right)_{W}\left(x_{0}, p_{0}\right) x_{t}^{(C l)} .
\end{aligned}
$$

Our goal would be to establish a relationship between Eq. (28) and the corresponding centroid correlation function.

We start by rewriting the exact $C_{A x}^{\text {Kubo }}(t)$ of Eq. (27) in a more convenient form:

$$
C_{A x}^{\mathrm{Kubo}}(t)=\frac{1}{\beta} \int_{0}^{\beta} d \lambda \operatorname{Tr}\left(\hat{A} e^{-\lambda \hat{H}} e^{i \hat{H} t / \hbar} \hat{x} e^{-i \hat{H} t / \hbar} e^{-(\beta-\lambda) \hat{H}}\right) .
$$

The integral over $\lambda$ is then discretized (the limit $P \rightarrow \infty$ will be reinforced at a later stage):

$$
\begin{aligned}
& C_{A x}^{\mathrm{Kubo}}(t) \\
& \quad=\frac{1}{P} \sum_{k=1}^{P} \operatorname{Tr}\left(\hat{A} e^{-k \beta / P \hat{H}} e^{i \hat{H} t / \hbar} \hat{x} e^{-i \hat{H} t / \hbar} e^{-(P-k) \beta / P \hat{H}}\right) .
\end{aligned}
$$

Each term in this sum can be related to a mixed real and imaginary time contour that corresponds to the following sequence (see Fig. 2): (1) Imaginary time propagation from 0 to $-i \hbar(\beta-\lambda) \rightarrow-i \hbar(P-k) \beta / P\left(e^{-(\beta-\lambda) \hat{H}}\right.$

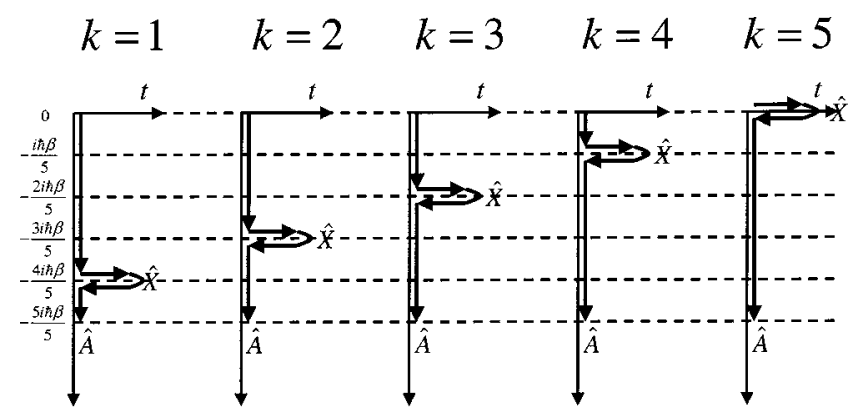

FIG. 2. A schematic view of the mixed imaginary and real time contours underlying Eq. (30). The picture corresponds to $P=5$, and each of the contours represents one contribution to the Kubo integral. The real and imaginary time axes are similar to these in Fig. 1. 


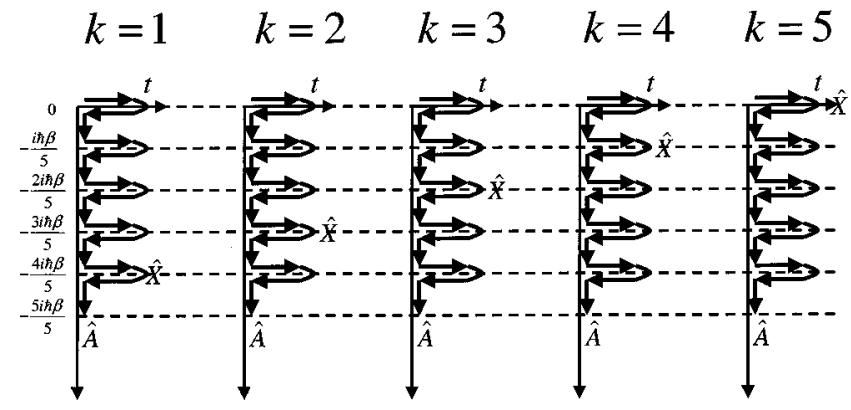

FIG. 3. A schematic view of the time contour underlying the path integral of Eq. (31). The picture corresponds to $P=5$, and each of the contours represents one contribution to the Kubo integral. The real- and imaginary-time axes are similar to these in Fig. 1.

$\left.\rightarrow e^{-(P-k) \beta \hat{H} / P}\right) ;(2)$ Forward real-time propagation from time 0 to time $t\left(e^{-i \hat{H} t / \hbar}\right)$; (3) Operating with the operator $\hat{x}$; (4) Backward real-time propagation from time $t$ to time 0 $\left(e^{i \hat{H} t / \hbar}\right) ;(5)$ Imaginary time propagation from $-i \hbar(\beta-\lambda)$ $\rightarrow-i \hbar(P-k) \beta / P$ to $-i \hbar \beta\left(e^{-\lambda \hat{H}} \rightarrow e^{-k \beta \hat{H} / P}\right)$; (6) Operating with $\hat{A}$. In order to perform the trace in Eq. (30), one has to perform this series of operations on each element of the basis of choice, and evaluate the overlap between the original and resulting states. The dominant contributions will obviously come from basis elements with nonvanishing overlaps.

In order to expose the relationship between LSC-IVR and centroid correlation functions, we found it useful to use a somewhat different time contour, which is nevertheless completely equivalent to the one described above. The new time contour involves a forward and backward real-time propagation at every point along the imaginary time axis $(\mathrm{cf}$. Fig. 3). More specifically, we replace each of the $e^{-\beta / P \hat{H}}$ factors, except for the one preceding $e^{i \hat{H} t / \hbar} \hat{x} e^{-i \hat{H} t / \hbar}$, by $e^{-\beta / P \hat{H}} e^{i \hat{H} t / \hbar} e^{-i \hat{H} t / \hbar}$ (this is obviously allowed since $e^{i \hat{H} t / \hbar} e^{-i \hat{H} t / \hbar}=\hat{I}$ ). Thus, Eq. (30) can now be put in the following form:

$$
\begin{aligned}
C_{A x}^{\mathrm{Kubo}}(t)= & \frac{1}{P} \sum_{k=1}^{P} \operatorname{Tr}\left[\hat{A}\left(e^{-\beta / P \hat{H}} e^{i \hat{H} t / \hbar} e^{-i \hat{H} t / \hbar}\right)^{k-1}\right. \\
& \times\left(e^{-\beta / P \hat{H}} e^{i \hat{H} t / \hbar} \hat{x} e^{-i \hat{H} t / \hbar}\right) \\
& \left.\times\left(e^{-\beta / P \hat{H}} e^{i \hat{H} t / \hbar} e^{-i \hat{H} t / \hbar}\right)^{P-k}\right] .
\end{aligned}
$$

Performing the trace in Eq. (31) in the position representation and inserting $3 P-1$ position closure relations between the various $e^{-\beta / P \hat{H}}, e^{i \hat{H} t / \hbar}$ and $e^{-i \hat{H} t / \hbar}$ factors, then yields:

$$
\begin{aligned}
C_{A x}^{\mathrm{Kubo}}(t)= & \int d x_{1, t} \ldots \int d x_{P, t} \int d x_{1,0}^{+} \ldots \int d x_{P, 0}^{+} \int d x_{1,0}^{-} \ldots \int d x_{P, 0}^{-}\left(\frac{1}{P} \sum_{k=1}^{P} x_{k, t}\right)\left\langle x_{1,0}^{+}\left|\hat{A} e^{-\beta / P \hat{H}}\right| x_{P, 0}^{-}\right\rangle\left\langle x_{P, 0}^{-}\left|e^{i \hat{H} t / \hbar}\right| x_{P, t}\right\rangle \\
& \times\left\langle x_{P, t}\left|e^{-i \hat{H} t / \hbar}\right| x_{P, 0}^{+}\right\rangle\left\langle x_{P, 0}^{+}\left|e^{-\beta / P \hat{H}}\right| x_{P-1,0}^{-}\right\rangle\left\langle x_{P-1,0}^{-}\left|e^{i \hat{H} t / \hbar}\right| x_{P-1, t}\right\rangle\left\langle x_{P-1, t}\left|e^{-i \hat{H} t / \hbar}\right| x_{P-1,0}^{+}\right\rangle \cdots\left\langle x_{2,0}^{+}\left|e^{-\beta / P \hat{H}}\right| x_{1,0}^{-}\right\rangle \\
& \times\left\langle x_{1,0}^{-}\left|e^{i \hat{H} t / \hbar}\right| x_{1, t}\right\rangle\left\langle x_{1, t}\left|e^{-i \hat{H} t / \hbar}\right| x_{1,0}^{+}\right\rangle .
\end{aligned}
$$

The identity $\sum_{k=1}^{P} x_{P-k+1, t}=\sum_{k=1}^{P} x_{k, t}$ has been utilized in order to obtain Eq. (32). Also, the notation adopted is based on using $\left|x_{k, 0}^{+}\right\rangle$for the initial state of the forward real-time propagation in the k-th branch, $\left|x_{k, 0}^{-}\right\rangle$as the final state of the backward real-time propagation in the k-th branch, and $\left|x_{k, t}\right\rangle$ for the final (initial) state of the forward (backward) realtime propagation in the $\mathrm{k}$-th branch.

We next express the product of all the real-time propagators in Eq. (32) in terms of the corresponding path integral expressions: ${ }^{112-114}$

$$
\begin{aligned}
\prod_{k=1}^{P}\left\langle x_{k, 0}^{-}\left|e^{i \hat{H} t / \hbar}\right| x_{k, t}\right\rangle\left\langle x_{k, t}\left|e^{-i \hat{H} t / \hbar}\right| x_{k, 0}^{+}\right\rangle \\
=\left(\frac{m}{2 \pi \hbar \epsilon}\right)^{N P} \prod_{k=1}^{P} \int d x_{k, 1}^{-} \cdots \int d x_{k, N-1}^{-} \\
\quad \times \int d x_{k, 1}^{+} \ldots \int d x_{k, N-1}^{+} \exp \left\{\frac{i}{\hbar} \sum_{k=1}^{P}\left(S_{k, N}^{+}-S_{k, N}^{-}\right)\right\},
\end{aligned}
$$

where,

$$
S_{k, N}^{ \pm}=\epsilon \sum_{j=0}^{N-1}\left[\frac{m}{2 \epsilon^{2}}\left(x_{k, j+1}^{ \pm}-x_{k, j}^{ \pm}\right)^{2}-V\left(x_{k, j}^{ \pm}\right)\right]
$$

(with the understanding that $x_{k, N}^{+}=x_{k, N}^{-}=x_{k, N}$ ). The path integrals in Eq. (33) were written in discrete form, such that $x_{k, t} \rightarrow x_{k, N}$ (the limit $N \rightarrow \infty$ will be reinforced at a later stage). It should also be noted that “ " $\prod_{k=1}^{P} \int d x_{k, 1}^{-} \cdots \int d x_{k, N-1}^{-} \int d x_{k, 1}^{+} \cdots \int d x_{k, N-1}^{+}$" stands for the $P$ forward-backward real-time integrations involved in Eq. (32) (the product " $\Pi_{k=1}^{P}$ " should not be applied to the integrand).

Substituting Eq. (33) into Eq. (32) then yields: 


$$
\begin{aligned}
C_{A x}^{\text {Kubo }}(t)= & \left(\frac{m}{2 \pi \hbar \epsilon}\right)^{N P} \prod_{k=1}^{P} \int d x_{k, 0}^{+} \cdots \int d x_{k, N-1}^{+} \int d x_{k, N} \int d x_{k, N-1}^{-} \cdots \int d x_{k, 0}^{-}\left(\frac{1}{P} \sum_{k=1}^{P} x_{k, N}\right)\left\langle x_{1,0}^{+}\left|\hat{A} e^{-\beta / P \hat{H}}\right| x_{P, 0}^{-}\right\rangle \\
& \times\left\langle x_{P, 0}^{+}\left|e^{-\beta / P \hat{H}}\right| x_{P-1,0}^{-}\right\rangle \cdots P\left(x_{2,0}^{+}\left|e^{-\beta / P \hat{H}}\right| x_{1,0}^{-}\right\rangle \exp \left\{\frac{i}{\hbar} \sum_{k=1}^{P}\left(S_{k, N}^{+}-S_{k, N}^{-}\right)\right\} .
\end{aligned}
$$

We next change the integration variables from $\left\{x_{k, 0}^{+}, \ldots, x_{k, N-1}^{+}, x_{k, N}, x_{k, N-1}^{-}, \ldots, x_{k, 0}^{-} \mid k=1, \ldots, P\right\}$ into $\left\{y_{k, 0}, \ldots, y_{k, N}, z_{k, 0}, \ldots, z_{k, N-1} \mid k=1, \ldots, P\right\}$, such that

$$
y_{k, j}=\frac{1}{2}\left(x_{k, j}^{+}+x_{k, j}^{-}\right), \quad z_{k, j}=\left(x_{k, j}^{+}-x_{k, j}^{-}\right)
$$

(we note that $y_{k, N}=x_{k, N}$ and $z_{k, N}=0$ ). We then linearize each of the forward-backward actions with respect to $z_{k, 0}, \ldots, z_{k, N-1}$ :

$$
\begin{aligned}
S_{k, N}^{+}-S_{k, N}^{-} \approx & \epsilon \sum_{j=0}^{N-1}\left[\frac{m}{\epsilon^{2}}\left(y_{k, j+1}-y_{k, j}\right)\left(z_{k, j+1}-z_{k, j}\right)\right. \\
& \left.-V^{\prime}\left(y_{k, j}\right) z_{k, j}\right] .
\end{aligned}
$$

Until this point, we followed a procedure similar to that in Sec. III. Furthermore, substituting Eq. (37) into Eq. (35), is bound to yield Eq. (28), due to the complete equivalence of the two time contours in Figs. 2 and 3. However, in the following steps, we divert from the procedure of Sec. III, with the goal of establishing the sought after relationship between LSC-IVR and centroid correlation functions. To this end, we first rewrite the sum of linearized forward-backward actions in terms of normal-mode coordinates:

$$
\begin{aligned}
\sum_{k=1}^{P}\left(S_{k, N}^{+}-S_{k, N}^{-}\right) & \\
\approx & -\epsilon \sum_{k=1}^{P}\left\{\left[\frac{m P}{\epsilon^{2}}\left(\tilde{y}_{k, 1}-\tilde{y}_{k, 0}\right)+\lambda_{k, 0}\right] \widetilde{z}_{k, 0}\right. \\
& \left.+\sum_{j=1}^{N-1}\left[\frac{m P}{\epsilon^{2}}\left(\tilde{y}_{k, j+1}-2 \tilde{y}_{k, j}+\tilde{y}_{k, j-1}\right)+\lambda_{k, j}\right] \widetilde{z}_{k, j}\right\},
\end{aligned}
$$

where

$$
\begin{aligned}
& \tilde{y}_{n, j}=\frac{1}{P} \sum_{k=1}^{P} U_{k, n} y_{k, j}, \quad \widetilde{z}_{n, j}=\frac{1}{P} \sum_{k=1}^{P} U_{k, n} z_{k, j}, \\
& y_{k, j}=\sum_{n=1}^{P} U_{k, n} \widetilde{y}_{n, j}, \quad z_{k, j}=\sum_{n=1}^{P} U_{k, n} \widetilde{z}_{n, j},
\end{aligned}
$$

$$
\begin{aligned}
& U_{k, 1}=1, \quad U_{k, P}=-(-1)^{k}, \\
& U_{k, 2 n-2}=\sqrt{2} \cos [2 \pi(k-1)(n-1) / P], \\
& U_{k, 2 n-1}=-\sqrt{2} \sin [2 \pi(k-1)(n-1) / P], \\
& U U^{\dagger}=U^{\dagger} U=P,
\end{aligned}
$$

and

$$
\lambda_{k, j}=\sum_{n=1}^{P} U_{n, k} V^{\prime}\left(y_{n, j}\right) .
$$

It should be noted that the time contour of Fig. 3 was actually introduced in anticipation of the transformation to normal mode coordinates. More specifically, based on this new time contour, each of the $P$ contributions to the Kubo integral is explicitly associated with the same action factor, $\exp \left\{i / \hbar \sum_{k=1}^{P}\left(S_{k, N}^{+}-S_{k, N}^{-}\right)\right\}$[cf. Eq. (35)]. A direct application of the original time contour would associate a different action factor, $\exp \left[i / \hbar\left(S_{k, N}^{+}-S_{k, N}^{-}\right)\right]$, with each of these contributions. Following linearization, it is straightforward to express $\exp \left\{i / \hbar \Sigma_{k=1}^{P}\left(S_{k, N}^{+}-S_{k, N}^{-}\right)\right\}$in terms of normal modes, which is not the case for $\Sigma_{k=1}^{P} \exp \left[i / \hbar\left(S_{k, N}^{+}-S_{k, N}^{-}\right)\right]$. Thus, although the two time contours are equivalent, the one employed (cf. Fig. 3 ) explicitly brings out the above mentioned symmetry of the integrand with respect to the the normal mode transformation.

We now change the integration variables from $y_{k, 1}, \ldots, y_{k, N}, z_{k, 0}, \ldots, z_{k, N-1}$ into $\widetilde{y}_{k, 1}, \ldots, \widetilde{y}_{k, N}, \widetilde{z}_{k, 0}, \ldots, \widetilde{z}_{k, N-1}$ (note that the integration variables $\left\{y_{k, 0} \mid k=1, \ldots, P\right\}$ remain in the primitive representation!). The integration over $\widetilde{z}_{k, 1}, \ldots, \widetilde{z}_{k, N-1}$ can be performed explicitly:

$$
\begin{array}{r}
\int d \widetilde{z}_{k, j} e^{-i / \hbar \epsilon\left(m P / \epsilon^{2}\left(\tilde{y}_{k, j+1}-2 \widetilde{y}_{k, j}+\tilde{y}_{k, j-1}\right)+\lambda_{k, j}\right) \tilde{z}_{k, j}} \\
=\frac{2 \pi \hbar}{\epsilon P} \delta\left(\frac{m}{\epsilon^{2}}\left(\widetilde{y}_{k, j+1}-2 \widetilde{y}_{k, j}+\widetilde{y}_{k, j-1}\right)+\frac{\lambda_{k, j}}{P}\right) .
\end{array}
$$

This leads to the following expression for $C_{A x}^{\mathrm{Kubo}}(t)$ :

$$
\begin{aligned}
C_{A x}^{\text {Kubo }}(t) \approx & P^{N P}\left(\frac{m}{2 \pi \hbar \epsilon}\right)^{N P}\left(\frac{2 \pi \hbar}{P \epsilon}\right)^{(N-1) P} \prod_{k=1}^{P} \int d \widetilde{z}_{k, 0} \int d y_{k, 0} \int d \widetilde{y}_{k, 1} \cdots \int d \widetilde{y}_{k, N} \widetilde{y}_{1, N}\left\langle y_{1,0}+z_{1,0} / 2\left|\hat{A} e^{-\beta / P \hat{H}}\right| y_{P, 0}-z_{P, 0} / 2\right\rangle \\
& \times\left\langle y_{P, 0}+z_{P, 0} / 2\left|e^{-\beta / P \hat{H}}\right| y_{P-1,0}-z_{P-1,0} / 2\right\rangle \cdots\left\langle y_{2,0}+z_{2,0} / 2\left|e^{-\beta / P \hat{H}}\right| y_{1,0}-z_{1,0} / 2\right\rangle \\
& \times \exp \left\{-\frac{i P}{\hbar}\left[\widetilde{z}_{1,0} \widetilde{p}_{1,0}+\cdots+\widetilde{z}_{P, 0} \widetilde{p}_{P, 0}\right]\right\} \prod_{j=1}^{N-1} \prod_{k=1}^{P} \delta\left(\frac{m}{\epsilon^{2}}\left(\widetilde{y}_{k, j+1}-2 \widetilde{y}_{k, j}+\widetilde{y}_{k, j-1}\right)+\frac{\lambda_{k, j}}{P}\right),
\end{aligned}
$$


uwhere the following identities were used:

$$
\begin{aligned}
& \tilde{y}_{1, N}=\frac{1}{P} \sum_{k=1}^{P} y_{k, N} \equiv \frac{1}{P} \sum_{k=1}^{P} x_{k, N}, \quad \tilde{p}_{k, 0}=\frac{m}{\epsilon}\left(\tilde{y}_{k, 1}-\tilde{y}_{k, 0}\right), \\
& \prod_{k=1}^{P} d z_{k, 0} \cdots d z_{k, N-1}=\left(P^{P / 2}\right)^{N} \prod_{k=1}^{P} d \tilde{z}_{k, 0} \cdots d \tilde{z}_{k, N-1}, \\
& \prod_{k=1}^{P} d y_{k, 1} \cdots d y_{k, N}=\left(P^{P / 2}\right)^{N} \prod_{k=1}^{P} d \tilde{y}_{k, 1} \cdots d \tilde{y}_{k, N} .
\end{aligned}
$$

It should be noted that the matrix elements in the integrand of Eq. (42) are given in terms of the primitive representation.

We next change the integration variables from $\tilde{y}_{k, 1}, \ldots, \tilde{y}_{k, N-1}$ into $f_{k, 1}, \ldots, f_{k, N-1}$, where

$$
f_{k, j}=\frac{m}{\epsilon^{2}}\left(\tilde{y}_{k, j+1}-2 \tilde{y}_{k, j}+\tilde{y}_{k, j-1}\right)+\frac{\lambda_{k, j}}{P},
$$

and explicitly integrate over these new integration variables. Imposing the limit $N \rightarrow \infty(\epsilon \rightarrow 0)$, such that $y_{k, N} \rightarrow y_{k, t}$, and using the identity in Eq. (24), then yields:

$$
\begin{aligned}
C_{A x}^{\text {Kubo }}(t) \approx & P^{P}\left(\frac{1}{2 \pi \hbar}\right)^{P} \prod_{k=1}^{P} \int d y_{k, 0} \int d \widetilde{y}_{k, t} \int d \widetilde{z}_{k, 0} \tilde{y}_{1, t}\left\langle y_{1,0}+z_{1,0} / 2\left|\hat{A} e^{-\beta / P \hat{H}}\right| y_{P, 0}-z_{P, 0} / 2\right\rangle \\
& \times\left\langle y_{P, 0}+z_{P, 0} / 2\left|e^{-\beta / P \hat{H}}\right| y_{P-1,0}-z_{P-1,0} / 2\right\rangle \cdots\left\langle y_{2,0}+z_{2,0} / 2\left|e^{-\beta / P \hat{H}}\right| y_{1,0}-z_{1,0} / 2\right\rangle \\
& \times \exp \left\{-\frac{i P}{\hbar}\left(\widetilde{z}_{1,0} \widetilde{p}_{1,0}+\cdots+\widetilde{z}_{P, 0} \widetilde{p}_{P, 0}\right)\right\} \prod_{k=1}^{P}\left|\frac{\partial \widetilde{p}_{k, 0}}{\partial \widetilde{y}_{k, t}}\right|,
\end{aligned}
$$

where the trajectory $\tilde{y}_{k}(t)$ satisfies the following classical-like equation of motion:

$$
m \frac{d^{2} \tilde{y}_{k, \tau}}{d \tau^{2}}=-\frac{\lambda_{k, \tau}}{P} .
$$

Equation (45) can then be put in the following form, upon transformation to IVR:

$$
\begin{aligned}
C_{A x}^{\mathrm{Kubo}}(t) \approx & P^{P}\left(\frac{1}{2 \pi \hbar}\right)^{P} \prod_{k=1}^{P} \int d y_{k, 0} \int d \widetilde{p}_{k, 0} \int d \widetilde{z}_{k, 0} \widetilde{y}_{1, t}\left\langle y_{1,0}+z_{1,0} / 2\left|\hat{A} e^{-\beta / P \hat{H}}\right| y_{P, 0}-z_{P, 0} / 2\right\rangle\left\langle y_{P, 0}+z_{P, 0} / 2\right| \\
& \times e^{-\beta / P \hat{H}}\left|y_{P-1,0}-z_{P-1,0} / 2\right\rangle \cdots P\left(y_{2,0}+z_{2,0} / 2\left|e^{-\beta / P \hat{H}}\right| y_{1,0}-z_{1,0} / 2\right\rangle \exp \left\{-\frac{i P}{\hbar}\left(\widetilde{z}_{1,0} \widetilde{p}_{1,0}+\cdots+\widetilde{z}_{P, 0} \widetilde{p}_{P, 0}\right)\right\} .
\end{aligned}
$$

Equation (46) dynamically couples the normal modes, $\tilde{y}_{1, \tau}, \ldots, \widetilde{y}_{P, \tau}$. In particular, the dynamics of the centroid, which corresponds to the first normal mode, $\tilde{y}_{1, \tau}$ $=\sum_{k=1}^{P} y_{k, \tau} / P$, is governed by the following equation:

$$
m \frac{d^{2} \tilde{y}_{1, \tau}}{d \tau^{2}}=-\frac{\lambda_{1, \tau}}{P}=-\frac{1}{P} \sum_{k=1}^{P} V^{\prime}\left(y_{k, \tau}\right),
$$

which obviously couples $\tilde{y}_{1, \tau}$ with the higher normal modes, $\tilde{y}_{2, \tau}, \ldots, \tilde{y}_{P, \tau}$. However, CMD, as well as other methods for approximating the centroid dynamics, ${ }^{29}$ are based on the implicit assumption that the centroid is decoupled from the higher normal modes. For example, the dynamics of $\tilde{y}_{1, \tau}$ in CMD is governed by the following classical-like equation of motion:

$$
m \frac{d^{2} \tilde{y}_{1, \tau}}{d \tau^{2}}=-V_{c m}^{\prime}\left(\tilde{y}_{1, \tau}\right) .
$$

Another example can be obtained if the following approximation is employed:

$$
\frac{1}{P} \sum_{k=1}^{P} V^{\prime}\left(y_{k, t}\right) \approx V^{\prime}\left(\frac{1}{P} \sum_{k=1}^{P} y_{k, t}\right)=V^{\prime}\left(\tilde{y}_{1, \tau}\right)
$$

in which case the dynamics is purely classical. It should also be noted that Eqs. (49) and (50) become exact for harmonic systems, where the centroid is always decoupled from the higher normal modes. These observations serve to explain why CMD is exact for harmonic systems and at the classical limit. However, they also suggest that recovering the centroid correlation function from Eq. (47), which is equivalent to the LSC-IVR approximation in Eq. (28), would require the additional assumption that the dynamics of $\tilde{y}_{1, \tau}$ is decoupled from that of the higher normal modes. It is important to note that the actual form of the decoupled centroid dynamics is irrelevant for the remainder of the derivation.

Assuming that $\tilde{y}_{1, \tau}$ is dynamically decoupled from the other normal modes, $\tilde{y}_{2, \tau}, \ldots, \widetilde{y}_{P, \tau}$, immediately implies that we can explicitly integrate over the momenta $\widetilde{p}_{2,0}, \ldots, \widetilde{p}_{P, 0}$ in Eq. (47):

$$
\begin{gathered}
\int d \widetilde{p}_{2,0} \cdots \int d \widetilde{p}_{P, 0} \exp \left\{-\frac{i P}{\hbar}\left(\widetilde{z}_{2,0} \widetilde{p}_{2,0}+\cdots+\widetilde{z}_{P, 0} \widetilde{p}_{P, 0}\right)\right\} \\
=\left(\frac{2 \pi \hbar}{P}\right)^{P-1} \delta\left(\widetilde{z}_{2,0}\right) \cdots \delta\left(\widetilde{z}_{P, 0}\right) .
\end{gathered}
$$

The resulting $\delta$ functions then make it possible to explicitly integrate over $\widetilde{z}_{2,0}, \ldots, \widetilde{z}_{P, 0}$, and set their values to zero in the 
result. As a consequence, $z_{k, 0}$ can be replaced by $\widetilde{z}_{1,0}[\mathrm{cf}$. Eq. (39)], thereby leading to the following approximation:

$$
\begin{aligned}
C_{A x}^{\text {Kubo }}(t) \approx & \frac{P}{2 \pi \hbar} \prod_{k=1}^{P} \int d y_{k, 0} \int d \widetilde{p}_{1,0} \int d \widetilde{z}_{1,0} \tilde{y}_{1, t} \\
& \times \exp \left\{-\frac{i P}{\hbar} \widetilde{z}_{1,0} \widetilde{p}_{1,0}\right\}\left\langle y_{1,0}+\widetilde{z}_{1,0} / 2\left|\hat{A} e^{-\beta / P \hat{H}}\right| y_{P, 0}\right. \\
& \left.-\widetilde{z}_{1,0} / 2\right\rangle\left\langle y_{P, 0}+\widetilde{z}_{1,0} / 2\left|e^{-\beta / P \hat{H}}\right| y_{P-1,0}\right. \\
& \left.-\widetilde{z}_{1,0} / 2\right\rangle \cdots\left\langle y_{2,0}+\widetilde{z}_{1,0} / 2\left|e^{-\beta / P \hat{H}}\right| y_{1,0}-\widetilde{z}_{1,0} / 2\right\rangle .
\end{aligned}
$$

In the next step, we rewrite the factor $\left\langle y_{1,0}\right.$ $\left.+\widetilde{z}_{1,0} / 2\left|\hat{A} e^{-\beta / P \hat{H}}\right| y_{P, 0}-\widetilde{z}_{1,0} / 2\right\rangle$ in the following form:

$$
\begin{aligned}
\left\langle y_{1,0}+\widetilde{z}_{1,0} / 2\left|\hat{A} e^{-\beta / P \hat{H}}\right| y_{P, 0}-\widetilde{z}_{1,0} / 2\right\rangle \\
=\int d y_{P+1}\left\langle y_{1,0}+\widetilde{z}_{1,0} / 2|\hat{A}| y_{P+1,0}+\widetilde{z}_{1,0} / 2\right\rangle \\
\quad \times\left\langle y_{P+1,0}+\widetilde{z}_{1,0} / 2\left|e^{-\beta / P \hat{H}}\right| y_{P, 0}-\widetilde{z}_{1,0} / 2\right\rangle .
\end{aligned}
$$

We then use the following relation (which becomes an identity at the limit $P \rightarrow \infty):{ }^{115}$

$$
\begin{aligned}
& \left\langle x_{1}\left|e^{-\beta / P \hat{H}}\right| x_{2}\right\rangle \\
& =\left(\frac{m P}{2 \pi \hbar^{2} \beta}\right)^{1 / 2} e^{-\beta\left[m P / 2 \beta^{2} \hbar^{2}\left(x_{1}-x_{2}\right)^{2}+1 / 2 P\left[V\left(x_{1}\right)+V\left(x_{2}\right)\right]\right]},
\end{aligned}
$$

in order to show that

$$
\begin{aligned}
\left\langle y_{P+1,0}+\widetilde{z}_{1,0} / 2\left|e^{-\beta / P \hat{H}}\right| y_{P, 0}-\widetilde{z}_{1,0} / 2\right\rangle \cdots\left\langle y_{2,0}+\widetilde{z}_{1,0} / 2\left|e^{-\beta / P \hat{H}}\right| y_{1,0}-\widetilde{z}_{1,0} / 2\right\rangle \\
=\left(\frac{m P}{2 \pi \hbar^{2} \beta}\right)^{P / 2} \exp \left\{-\frac{m P}{2 \beta \hbar^{2}}\left[P \widetilde{z}_{1,0}^{2}+2 \widetilde{z}_{1,0}\left(y_{P+1,0}-y_{1,0}\right)+\left(y_{P+1,0}-y_{P, 0}\right)^{2}+\ldots+\left(y_{2,0}-y_{1,0}\right)^{2}\right]\right. \\
\quad+\frac{1}{P}\left[\frac{1}{2} V\left(y_{P+1,0}+\widetilde{z}_{1,0} / 2\right)+\frac{1}{2}\left[V\left(y_{P, 0}+\widetilde{z}_{1,0} / 2\right)+V\left(y_{P, 0}-\widetilde{z}_{1,0} / 2\right)\right]\right. \\
\left.\left.\quad+\ldots+\frac{1}{2}\left[V\left(y_{2,0}+\widetilde{z}_{1,0} / 2\right)+V\left(y_{2,0}-\widetilde{z}_{1,0} / 2\right)\right]+\frac{1}{2} V\left(y_{1,0}-\widetilde{z}_{1,0} / 2\right)\right]\right\} .
\end{aligned}
$$

We then note that at the limit $P \rightarrow \infty$, the Gaussian factor $\exp \left[-\left(m P^{2} / 2 \beta \hbar^{2}\right) \widetilde{z}_{1,0}^{2}\right]$ becomes very narrow, such that $V\left(y_{k, 0}\right.$ $\left.\pm \widetilde{z}_{1,0} / 2\right)$ in Eq. (55) and $\left\langle y_{1,0}+\widetilde{z}_{1,0} / 2|\hat{A}| y_{P+1,0}+\widetilde{z}_{1,0} / 2\right\rangle$ in Eq. (53) can be replaced by $V\left(y_{k, 0}\right)$ and $\left\langle y_{1,0}|\hat{A}| y_{P+1,0}\right\rangle$, respectively. It should be noted that this argument does not apply to the factors $\exp \left\{-\left(m P / \beta \hbar^{2}\right) \widetilde{z}_{1,0}\left(y_{P+1,0}-y_{1,0}\right)\right\}$ in Eq. $(55)$, and $\exp \left\{-(i P / \hbar) \widetilde{z}_{1,0} \widetilde{p}_{1,0}\right\}$ in Eq. (52), since the corresponding exponents are proportional to $P \widetilde{z}_{1,0}$. Thus, one is left with the evaluation of the following Gaussian integral:

$$
\begin{aligned}
& \int d \widetilde{z}_{1,0} \exp \left\{-\frac{m P^{2}}{2 \beta \hbar^{2}} \widetilde{z}_{1,0}^{2}-\frac{i}{\hbar} P \widetilde{p}_{1,0} \widetilde{z}_{1,0}-\frac{m P}{\beta \hbar^{2}}\left(y_{P+1,0}-y_{1,0}\right) \widetilde{z}_{1,0}\right\} \\
& =\left(\frac{2 \pi \hbar^{2} \beta}{m P^{2}}\right)^{1 / 2} \exp \left\{-\frac{\beta}{2 m} \widetilde{p}_{1,0}^{2}+\frac{i}{\hbar}\left(y_{P+1,0}-y_{1,0}\right) \widetilde{p}_{1,0}+\frac{m}{2 \beta \hbar^{2}}\left(y_{P+1,0}-y_{1,0}\right)^{2}\right\} .
\end{aligned}
$$

Incorporating the results from the previous paragraph into Eq. (52) transforms it into:

$$
\begin{aligned}
C_{A x}^{\text {Kubo }}(t) \approx & \frac{1}{2 \pi \hbar}\left(\frac{2 \pi \hbar^{2} \beta}{m}\right)^{1 / 2}\left(\frac{m P}{2 \pi \hbar^{2}}\right)^{P / 2} \int d \widetilde{p}_{1,0} \int d y_{1,0} \ldots \int d y_{P+1,0} \tilde{y}_{1, t}\left\langle y_{1,0}|\hat{A}| y_{P+1,0}\right\rangle \exp \left\{-\frac{\beta \widetilde{p}_{1,0}^{2}}{2 m}\right\} \\
& \times \exp \left\{\frac{m}{2 \beta \hbar^{2}}\left(y_{P+1,0}-y_{1,0}\right)^{2}\right\} \exp \left\{\frac{i}{\hbar}\left(y_{P+1,0}-y_{1,0}\right) \tilde{p}_{1,0}\right\} \exp \left\{-\frac{m P}{2 \beta \hbar^{2}}\left[\left(y_{P+1,0}-y_{P, 0}\right)^{2}+\cdots+\left(y_{2,0}-y_{1,0}\right)^{2}\right]\right\} \\
& \times \exp \left\{-\frac{\beta}{P}\left[\frac{1}{2} V\left(y_{1,0}\right)+V\left(y_{2,0}\right)+\ldots+V\left(y_{P, 0}\right)+\frac{1}{2} V\left(y_{P+1,0}\right)\right]\right\}
\end{aligned}
$$

Equation (57) is our final result. It can be shown to coincide with the following centroid correlation function:

$$
\frac{1}{2 \pi \hbar} \int d x_{c} \int d p_{c} \rho_{c}\left(x_{c}, p_{c}\right) A_{c}\left(x_{c}, p_{c}\right) x_{c}(t),
$$

[the proof for the equivalence of Eqs. (57) and (58) is presented in the Appendix]. It should be noted that the time evolution of $x_{c}(t)$ in Eq. (58) remains undefined, except for the assumption that it is decoupled from that of the higher normal modes. Several approximations for propagating $x_{c}(t)$ have been proposed in the past, including classical mechan- 
ics, CMD, and other types of approximated centroid dynamics. ${ }^{29}$ The relationship between LSC-IVR and centroid correlation functions developed herein is not limited to any one of these approximations, since they are all based on the implicit assumption that the centroid dynamics is decoupled from that of the higher normal modes.

\section{CONCLUSION}

The main contributions of this paper can be summarized as follows:

- We have shown that the LSC-IVR approximation for the two-time quantum-mechanical correlation function can be directly obtained by linearizing the forwardbackward action in the corresponding exact pathintegral expression.

- We established a relationship between the LSC-IVR and centroid correlation functions, and showed that the latter can be obtained from the former if the dynamics of the centroid is assumed to be decoupled from that of the higher normal modes.

A schematic chart that maps the significance of these new results and their relationship to results obtained by other workers is given in Fig. 4.

The second contribution deserves special attention, since it provides a bridge between an imaginary-time (centroid) approach and a real-time (SC) approach. It should be noted that although the exact real-time dynamics of centroid symbols can be formulated ${ }^{28}$ [cf. Eq. (8)], practical implementations, such as CMD, have been based on the notion that this real-time information can be obtained from imaginary-time simulations. ${ }^{29}$ The present analysis does not imply that this strategy is inferior to the SC approach. Rather, it points to another implicit assumption, namely that the centroid dynamics is decoupled from that of the other normal modes. Our main observation is that this seems to be the only assumption that distinguishes the centroid approach from the LSC-IVR approach. It also seems to indicate that explicitly accounting for the coupling between the centroid and the other normal modes may improve the accuracy of the centroid approach.

The picture that emerges from this analysis is that despite the common SC flavor of LSC-IVR and centroid methods, they differ in the way they treat the centroid dynamics. Practical implementations of the centroid-based approach, such as CMD, are based on the implicit assumption that the centroid dynamics is decoupled from that of the higher normal modes. LSC-IVR does not require us to make this assumption, and is therefore less approximate in this respect. It should be noted however, that the actual dynamics in LSCIVR is classical, whereas methods like CMD can partly account for quantum dynamical effects via "quantumcorrected" centroid force fields. This may imply that centroid-based methods can actually capture quantummechanical coherent effects for a longer period of time. Moreover, the fact that LSC-IVR is less approximate has its price, since sampling the Wigner distribution of a general anharmonic many-body system is usually more computationally demanding than sampling the classical-like centroid dis-

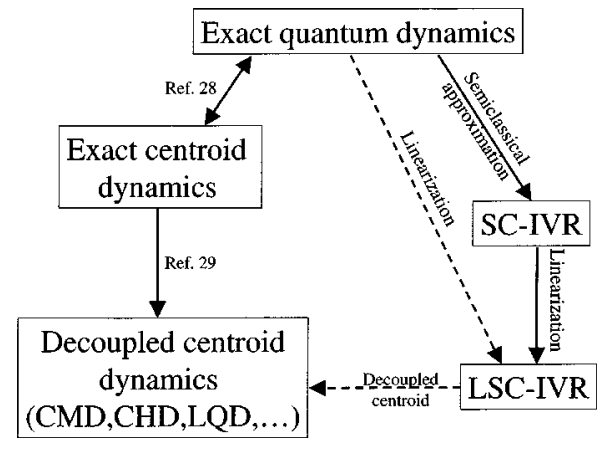

FIG. 4. Links between semiclassical, centroid and exact quantum methods. Arrows represent approximations (the arrow points toward the more approximated method). Double arrows represent equivalence. The solid lines represent previously derived links, while the dashed lines represent new links derived in the present paper.

tribution. Thus, in cases where there is a choice between the two methods, the centroid approach is the more costeffective route, provided that decoupled centroid dynamics is a valid assumption. Barrier crossing rate constants, where LSC-IVR and CMD were found to give similar and accurate predictions when tested against the same benchmark, ${ }^{45-47,111}$ appears to be such a case. However, we have recently found that the opposite situation arises in the case of vibrational energy relaxation rate constants, where LSC-IVR seems to be significantly more accurate than CMD. ${ }^{116}$ It is hoped that the better understanding of the relationship between SC and centroid methods established in the present paper, would help guide future attempts to improve their efficiency and accuracy, as well as give rise to a better understanding of the different roles played by quantum mechanics in a variety of condensed phase systems.

\section{ACKNOWLEDGMENTS}

The work reported in this paper was partially supported by the National Science Foundation FOCUS Center, Grant No. 0114336.

\section{APPENDIX: THE CENTROID CORRELATION FUNCTION}

In this Appendix, we prove that Eqs. (57) and (58) are identical. To this end, consider the centroid correlation function of Eq. (58):

$$
C_{A x}^{C}(t)=\int d x_{c} \int d p_{c} \rho_{c}\left(x_{c}, p_{c}\right) A_{c}\left(x_{c}, p_{c}\right) x_{c}(t),
$$

where the dynamics of $x_{c}(t)$ can remain unspecified for the purpose of the following derivation. Substituting Eq. (3) for $A_{c}\left(x_{c}, p_{c}\right)$, we obtain:

$$
C_{A x}^{C}(t)=\int d x_{c} \int d p_{c} \operatorname{Tr}\left[\phi_{c}\left(x_{c}, p_{c}\right) \hat{A}\right] x_{c}(t) .
$$

Using Eqs. (13) and (14) of Ref. 29, one can show that 


$$
\begin{aligned}
\operatorname{Tr}\left[\phi_{c}\left(x_{c}, p_{c}\right) \hat{A}\right]= & \int d x_{1} \int d x_{P+1}\left\langle x_{P+1}\left|\phi_{c}\left(x_{c}, p_{c}\right)\right| x_{1}\right\rangle\left\langle x_{1}|\hat{A}| x_{P+1}\right\rangle \delta\left(x_{c}-\frac{1}{P} \sum_{k=1}^{P} x_{k}\right) \\
= & \left(\frac{2 \pi \hbar^{2} \beta}{m}\right)^{1 / 2}\left(\frac{m P}{2 \pi \hbar^{2}}\right)^{P / 2} \int d x_{1} \cdots \int d x_{P+1}\left\langle x_{1}|\hat{A}| x_{P+1}\right\rangle e^{-\beta p_{c}^{2} / 2 m} e^{m P / 2 \beta \hbar^{2}\left(x_{P+1}-x_{1}\right)^{2}} e^{i / \hbar\left(x_{P+1}-x_{1}\right) p_{c}} \\
& \times e^{-m P / 2 \beta \hbar^{2}\left[\left(x_{P+1}-x_{P}\right)^{2}+\cdots+\left(x_{2}-x_{1}\right)^{2}\right]} e^{-\beta / P\left[1 / 2 V\left(x_{1}\right)+V\left(x_{2}\right)+\ldots V\left(x_{P}\right)+1 / 2 V\left(x_{P+1}\right)\right]} .
\end{aligned}
$$

Substituting Eq. (A3) back into Eq. (A2) yields:

$$
\begin{aligned}
C_{A x}^{C}(t)= & \frac{1}{2 \pi \hbar}\left(\frac{2 \pi \hbar^{2} \beta}{m}\right)^{1 / 2}\left(\frac{m P}{2 \pi \hbar^{2}}\right)^{P / 2} \int d p_{c} \int d x_{1} \cdots \int d x_{P+1}\left\langle x_{1}|\hat{A}| x_{P+1}\right\rangle e^{-\beta p_{c}^{2} / 2 m} e^{m / 2 \beta \hbar^{2}\left(x_{P+1}-x_{1}\right)^{2}} e^{i / \hbar\left(x_{P+1}-x_{1}\right) p_{c}} \\
& \times e^{-m P / 2 \beta \hbar^{2}\left[\left(x_{P+1}-x_{P}\right)^{2}+\cdots+\left(x_{2}-x_{1}\right)^{2}\right]} e^{-\beta / P\left[1 / 2 V\left(x_{1}\right)+V\left(x_{2}\right)+\ldots+V\left(x_{P}\right)+1 / 2 V\left(x_{P+1}\right)\right]} .
\end{aligned}
$$

Equation (A4) is identical to Eq. (57) [it should be noted that the variables $x_{1}, \ldots, x_{P+1}, p_{c}$ and $x_{c}(t)$ in Eq. (A4) are denoted by $y_{1,0}, \ldots, y_{P+1,0}, \widetilde{p}_{1,0}$, and $\tilde{y}_{1, t}$ in Eq. (57), respectively].

${ }^{1}$ D. A. McQuarrie, Statistical Mechanics (Harper and Row, New York, 1976).

${ }^{2}$ R. Kubo, M. Toda, and N. Hashitsume, Statistical Physics IINonequilibrium Statistical Mechanics (Springer-Verlag, Berlin, 1983).

${ }^{3}$ D. Chandler, Introduction to Modern Statistical Mechanics (Oxford University Press, New York, 1987).

${ }^{4}$ M. P. Allen and D. J. Tildesley, Computer Simulation of Liquids (Clarendon, Oxford, 1987).

${ }^{5}$ B. J. Berne, G. Ciccotti, and D. F. Coker, Eds., Classical and Quantum Dynamics in Condensed Phase Simulations (World Scientific, New Jersey, 1998).

${ }^{6}$ S. Mukamel, Principles of Nonlinear Optical Spectroscopy (Oxford, New York, 1995).

${ }^{7}$ N. Makri, Annu. Rev. Phys. Chem. 50, 167 (1999).

${ }^{8}$ G. D. Billing, Chem. Phys. Lett. 30, 391 (1975).

${ }^{9}$ G. D. Billing, J. Chem. Phys. 99, 5849 (1993).

${ }^{10}$ J. C. Tully and R. K. Preston, J. Chem. Phys. 55, 562 (1971).

${ }^{11}$ J. C. Tully, J. Chem. Phys. 93, 1061 (1990).

${ }^{12}$ P. J. Kuntz, J. Chem. Phys. 95, 141 (1991).

${ }^{13}$ A. I. Krylov et al., J. Chem. Phys. 104, 3651 (1996).

${ }^{14}$ J. Poulsen and P. J. Rossky, J. Chem. Phys. 115, 8014 (2001).

${ }^{15}$ K. Yamashita and W. H. Miller, J. Chem. Phys. 82, 5475 (1985).

${ }^{16}$ E. Gallicchio and B. J. Berne, J. Chem. Phys. 105, 7064 (1996).

${ }^{17}$ E. Gallicchio, S. A. Egorov, and B. J. Berne, J. Chem. Phys. 109, 7745 (1998).

${ }^{18}$ S. A. Egorov, E. Gallicchio, and B. J. Berne, J. Chem. Phys. 107, 9312 (1997).

${ }^{19}$ G. Krilov and B. J. Berne, J. Chem. Phys. 111, 9147 (1999).

${ }^{20}$ E. Rabani, G. Krilov, and B. J. Berne, J. Chem. Phys. 112, 2605 (2000).

${ }^{21}$ E. Sim, G. Krilov, and B. Berne, J. Phys. Chem. A 105, 2824 (2001).

${ }^{22}$ J. Cao and G. A. Voth, J. Chem. Phys. 100, 5093 (1994).

${ }^{23}$ J. Cao and G. A. Voth, J. Chem. Phys. 100, 5106 (1994).

${ }^{24}$ J. Cao and G. A. Voth, J. Chem. Phys. 101, 6157 (1994).

${ }^{25}$ J. Cao and G. A. Voth, J. Chem. Phys. 101, 6168 (1994).

${ }^{26}$ J. Cao and G. A. Voth, J. Chem. Phys. 101, 6184 (1994).

${ }^{27}$ G. A. Voth, Adv. Chem. Phys. 93, 135 (1996).

${ }^{28}$ S. Jang and G. A. Voth, J. Chem. Phys. 111, 2357 (1999).

${ }^{29}$ S. Jang and G. A. Voth, J. Chem. Phys. 111, 2371 (1999).

${ }^{30}$ D. R. Reichman, P.-N. Roy, S. Jang, and G. A. Voth, J. Chem. Phys. 113, 919 (2000).

${ }^{31}$ A. Calhoun, M. Pavese, and G. A. Voth, Chem. Phys. Lett. 262, 415 (1996).

${ }^{32}$ U. W. Schmitt and G. A. Voth, J. Chem. Phys. 111, 9361 (1999).

${ }^{33}$ M. Pavese and G. A. Voth, Chem. Phys. Lett. 249, 231 (1996).

${ }^{34}$ K. Kinugawa, P. B. Moore, and M. L. Klein, J. Chem. Phys. 106, 1154 (1997).

${ }^{35}$ K. Kinugawa, P. B. Moore, and M. L. Klein, J. Chem. Phys. 109, 610 (1998).
${ }^{36}$ K. Kinugawa, Chem. Phys. Lett. 292, 454 (1998).

${ }^{37}$ M. Pavese, D. R. Bernard, and G. A. Voth, Chem. Phys. Lett. 300, 93 (1999).

${ }^{38}$ S. Jang, Y. Pak, and G. A. Voth, J. Phys. Chem. A 103, 10289 (1999).

${ }^{39}$ R. Ramirez, T. Lopez-Ciudad, and J. C. Noya, Phys. Rev. Lett. 81, 3303 (1998).

${ }^{40}$ R. Ramirez and T. Lopez-Ciudad, Phys. Rev. Lett. 83, 4456 (1999).

${ }^{41}$ R. Ramirez and T. Lopez-Ciudad, J. Chem. Phys. 111, 3339 (1999).

${ }^{42}$ T. Lopez-Ciudad and R. Ramirez, J. Chem. Phys. 113, 10849 (2000).

${ }^{43}$ J. Poulsen, S. R. Keiding, and P. J. Rossky, Chem. Phys. Lett. 336, 488 (2001).

${ }^{44}$ J. Poulsen and P. J. Rossky, J. Chem. Phys. 115, 8024 (2001).

${ }^{45}$ E. Geva, Q. Shi, and G. A. Voth, J. Chem. Phys. 115, 9209 (2001).

${ }^{46}$ Q. Shi and E. Geva, J. Chem. Phys. 116, 3223 (2002).

${ }^{47}$ H. Wang, X. Sun, and W. H. Miller, J. Chem. Phys. 108, 9726 (1998).

${ }^{48}$ E. Pollak and J. Liao, J. Chem. Phys. 108, 2733 (1998).

${ }^{49}$ W. H. Miller, Adv. Chem. Phys. 25, 69 (1974).

${ }^{50}$ W. H. Miller, J. Chem. Phys. 53, 3578 (1970).

${ }^{51}$ M. F. Herman and E. Kluk, Chem. Phys. 91, 27 (1984).

${ }^{52}$ E. J. Heller, J. Chem. Phys. 94, 2723 (1981).

${ }^{53}$ K. G. Kay, J. Chem. Phys. 100, 4377 (1994).

${ }^{54}$ M. Ovchinnikov and V. A. Apkarian, J. Chem. Phys. 105, 10312 (1996).

${ }^{55}$ M. Ovchinnikov and V. A. Apkarian, J. Chem. Phys. 108, 2277 (1998).

${ }^{56}$ X. Sun and W. H. Miller, J. Chem. Phys. 106, 916 (1997).

${ }^{57}$ N. Makri and K. Thompson, Chem. Phys. Lett. 291, 101 (1998).

${ }^{58}$ W. H. Miller, Faraday Discuss. 110, 1 (1998).

${ }^{59}$ J. S. Shao and N. Makri, J. Phys. Chem. A 103, 7753 (1999).

${ }^{60}$ K. Thompson and N. Makri, Phys. Rev. E 59, R4729 (1999).

${ }^{61}$ O. Kühn and N. Makri, J. Phys. Chem. A 103, 9487 (1999).

${ }^{62}$ H. Wang, M. Thoss, and W. H. Miller, J. Chem. Phys. 112, 47 (2000).

${ }^{63}$ M. Ovchinnikov, V. A. Apkarian, and G. A. Voth, J. Chem. Phys. 184, $7130(2001)$

${ }^{64}$ W. H. Miller, J. Phys. Chem. A 105, 2942 (2001).

${ }^{65}$ N. Makri and W. H. Miller, J. Chem. Phys. 116, 9207 (2002).

${ }^{66}$ B. J. Berne and D. Thirumalai, Annu. Rev. Phys. Chem. 37, 401 (1986).

${ }^{67}$ D. M. Ceperley, Rev. Mod. Phys. 67, 279 (1995).

${ }^{68}$ J. H. V. Vleck, Proc. Natl. Acad. Sci. U.S.A. 14, 178 (1928).

${ }^{69}$ M. C. Gutzwiller, J. Math. Phys. 8, 1979 (1967).

${ }^{70}$ M. C. Gutzwiller, J. Math. Phys. 12, 343 (1971).

${ }^{71}$ M. C. Gutzwiller, Chaos in Classical and Quantum Mechanics (SpringerVerlag, Berlin, 1990).

${ }^{72}$ R. G. Littlejohn, J. Stat. Phys. 68, 7 (1992).

${ }^{73}$ P. Pechukas, Phys. Rev. 181, 174 (1969).

${ }^{74}$ S. Levit and U. Smilansky, Ann. Phys. (N.Y.) 108, 165 (1977).

${ }^{75}$ S. Levit, K. Mohring, U. Smilansky, and T. Dreyfus, Ann. Phys. (N.Y.) 114, 223 (1978).

${ }^{76}$ V. P. Maslov and M. V. Fedoriouk, Semi-Classical Approximation in Quantum Mechanics (Reidel, Boston, 1981).

${ }^{77}$ W. H. Miller, J. Chem. Phys. 95, 9428 (1991).

${ }^{78}$ E. J. Heller, J. Chem. Phys. 95, 9431 (1991).

${ }^{79}$ E. Kluk, M. F. Herman, and H. L. Davis, J. Chem. Phys. 84, 326 (1986).

${ }^{80}$ K. G. Kay, J. Chem. Phys. 101, 2250 (1994).

${ }^{81}$ D. Provost and P. Brumer, Phys. Rev. Lett. 74, 250 (1995).

${ }^{82}$ S. Garashchuk and D. Tannor, Chem. Phys. Lett. 262, 477 (1996). 
${ }^{83}$ S. Keshavamurthy and W. H. Miller, Chem. Phys. Lett. 218, 183 (1994).

${ }^{84}$ B. W. Spath and W. H. Miller, J. Chem. Phys. 104, 95 (1996).

${ }^{85}$ J. D. Doll, D. L. Freeman, and T. L. Beck, Adv. Chem. Phys. 78, 61 (1990).

${ }^{86}$ V. S. Filinov, Nucl. Phys. B 271, 717 (1986).

${ }^{87}$ N. Makri and W. H. Miller, Chem. Phys. Lett. 139, 10 (1987).

${ }^{88}$ A. R. Walton and D. E. Manolopoalos, Mol. Phys. 87, 961 (1996).

${ }^{89}$ X. Sun and W. H. Miller, J. Chem. Phys. 108, 8870 (1998).

${ }^{90}$ X. Sun and W. H. Miller, J. Chem. Phys. 110, 6635 (1999).

${ }^{91}$ H. Wang, D. E. Manolopoulos, and W. H. Miller, J. Chem. Phys. 115, 6317 (2001).

${ }^{92}$ X. Sun, H. Wang, and W. H. Miller, J. Chem. Phys. 109, 4190 (1998).

${ }^{93}$ X. Sun, H. Wang, and W. H. Miller, J. Chem. Phys. 109, 7064 (1998).

${ }^{94}$ H. Wang, X. Song, D. Chandler, and W. H. Miller, J. Chem. Phys. 110, 4828 (1999).

${ }^{95}$ W. H. Miller, J. Phys. Chem. A 103, 9384 (1999).

${ }^{96}$ J. S. Shao, J. L. Liao, and E. Pollak, J. Chem. Phys. 108, 9711 (1998).

${ }^{97}$ J. L. Liao and E. Pollak, Chem. Phys. 268, 295 (2001).

${ }^{98}$ M. Hillery, R. F. O'Connell, M. O. Scully, and E. P. Wigner, Phys. Rep. 106, 121 (1984).

${ }^{99}$ K. Thompson and N. Makri, J. Chem. Phys. 13, 1343 (1999).

${ }^{100}$ K. Thompson and N. Makri, J. Chem. Phys. 59, R4729 (1999).
${ }^{101}$ V. S. Batista et al., J. Chem. Phys. 110, 3736 (1999).

${ }^{102}$ M. Thoss, H. Wang, and W. H. Miller, J. Chem. Phys. 114, 9220 (2001).

${ }^{103}$ F. Grossmann, Chem. Phys. Lett. 262, 470 (1996).

${ }^{104}$ M. Thoss and W. H. Miller, J. Chem. Phys. 112, 10282 (2000).

${ }^{105}$ E. A. Coronado, J. Xing, and W. H. Miller, Chem. Phys. Lett. 349, 521 (2001).

${ }^{106}$ A. R. Walton and D. E. Manolopoalos, Chem. Phys. Lett. 244, 448 (1995).

${ }^{107}$ B. W. Spath and W. H. Miller, Chem. Phys. Lett. 262, 486 (1996).

${ }^{108}$ V. S. Batista and W. H. Miller, J. Chem. Phys. 108, 498 (1998).

${ }^{109}$ M. L. Brewer, J. S. Hulme, and D. E. Manolopoulos, J. Chem. Phys. 106, 4832 (1997).

${ }^{110}$ H. Wang et al., J. Chem. Phys. 114, 2562 (2001).

${ }^{111}$ M. Topaler and N. Makri, J. Chem. Phys. 101, 7500 (1994).

${ }^{112}$ L. S. Schulman, Techniques and Applications of Path Integration (Wiley, New York, 1981)

${ }^{113}$ R. P. Feynman and A. R. Hibbs, Quantum Mechanics and Path Integrals (McGraw-Hill, New York, 1965).

${ }^{114} \mathrm{H}$. Kleinert, Path Integrals in Quantum Mechanics, Statistics and Polymer Physics (World Scientific, New Jersey, 1995).

${ }^{115}$ R. P. Feynman, Statistical Mechanics (Benjamin, New York, 1972).

${ }^{116} \mathrm{Q}$. Shi and E. Geva (unpublished). 